\title{
PROTEIN AND RIBONUCLEIC ACID IN THE GROWTH OF FERN GAMETOPHYTES
}

\begin{abstract}
TN many Polypodiaceae, the germinating spore 1 yields a filamentous protonema and rhizoid cell, that is, a 'one-dimensional' growth. After a certain time-lapse, the growing protonema begins to divide by longitudinal walls also, thereby yielding a platelike structure, or 'two-dimensional' system. This leads on to the typical heart-shaped prothallus or gametophyte. Y. Hotta (Jap. J. Bot., 17, $214 ;$ 1960) has now contributed now facts about the role of protein and ribonucleic acid in the differentiation of the gametophyte of Dryopteris erythrosora.

Estimation of the protein contents during the course of development clearly indicates that whenever the two-dimensional growth takes place, a sharp increase in protein concentration in the cell can be observed, whereas in the course of the primary onedimensional growth, protein concentration is gradually lowered. The two-dimensional growth can be specifically inhibited by some amino-acid analogues such as ethionine and 5-methyltryptophan, and is converted to the 'artificial' one-dimensional growth.
\end{abstract}

The same selective interference of the twodimensional growth is also brought about by 8 -azaguanine, the effective inhibitor of the nucleic acid synthesis. This inhibition can be alleviated by adding corresponding amino-acids or guanine, respectively. Regardless of the nature of the effective inhibitors used, the selective inhibition is always accompanied by an immediate cessation of increase in the protein concentration followed by its gradual decrease. The author has also shown that ribonucleic acid from the two-dimensional prothallium possesses a nucleotide composition distinct from that in the one-dimensional growth. Ribonucleic acid found in the latter, induced artificially by 8-azaguanine, has been shown to have practically the same nucleotide composition as that of the primary one-dimensional growth. Accordingly, it has been tentatively inferred that a special ribonucleic acid fraction which is sensitive to the action of 8-aza. guanine is closely related to the two-dimensional growth.

\section{VIBRATIONALLY EXCITED NITRIC OXIDE PRODUCED IN THE FLASH PHOTOLYSIS OF NITROSYL CHLORIDE}

\author{
By Dr. N. BASCO and Prof. R. G. W. NORRISH, F.R.S. \\ Department of Physical Chemistry, University of Cambridge
}

\begin{abstract}
W HENEVER a molecule dissociates by absorbing radiation of shorter wave-length than is required from the known strength of the bond broken, the problem arises of the distribution of the excess energy among the fragments. Frequently it can be shown that one or more of these fragments are produced in an electronically excited state, but, in general, there is some energy which must appear in other forms. For a diatomic molecule, it follows that the atoms are produced with excess kinetic energy, but where a polyatomic molecule is concerned, there is the possibility that one or more of the products are produced vibrationally or rotationally excited in the ground electronic state. The present communication describes what seems to be the only direct experimental evidence that these possibilities are, in fact, realized.

In the flash photolysis of sulphur trioxide, Norrish and Oldershaw ${ }^{1}$ observed the presence of vibrationally excited SO with $v^{\prime \prime}<3$, but, though it was shown that the effect was not thermal, it was not possible to decide whether the excited molecule was produced by primary photodissociation or by secondary reactions, that is :
\end{abstract}

or

$$
\mathrm{SO}_{3}+h \nu \rightarrow \mathrm{SO}^{*}+\mathrm{O}_{2}
$$

followed by

$$
\mathrm{SO}_{3}+h \nu \rightarrow \mathrm{SO}_{2}+\mathrm{O}\left({ }^{\prime} D\right)
$$$$
\mathrm{O}\left({ }^{\prime} D\right)+\mathrm{SO}_{2} \rightarrow \mathrm{SO}^{*}+\mathrm{O}_{2}
$$

Similarly, in the flash photolysis of nitrogen dioxide, Basco and Norrish' observed the first vibrationally excited level of nitric oxide, but again it was not possible to decide whether it is a primary or secondary product, in the latter case derived from:

$$
\mathrm{O}+\mathrm{NO}_{2} \rightarrow \mathrm{NO}^{*}+\mathrm{O}_{2}
$$

We have now studied the flash photolysis of nitrosyl chloride in the presence of a great excess of inert gas to ensure isothermal conditions and have demonstrated the presence of very highly vibretionally excited nitric oxide. Many absorption bands in the region 2100-4000 $\AA$. have been observed and it has been possible to assign most of them to transitions from the ground state of NO $\left(X^{2} \Gamma\right)$ to the $A^{2} \Sigma$, $B^{2} \Pi, C^{2} \Pi$ and $D^{2} \Sigma$ excited states and thus to demonstrate that all vibrational levels of the ground state up to $v^{\prime \prime}=10$ are populated. Some bands remain unassigned or only tentatively so, but it seems likely that these also will be found to correspond to transitions from vibrationally excited levels of the ground electronic state of NO. The decay of the excited molecules is very rapid and those in the higher vibrational levels are only present in appreciable concentrations during the photolytic flash.

Nitrosyl chloride can be decomposed by light of wave-length below about $6400 \AA .^{3}$ and, even at this wave-length, there is a slight excess of energy over that required to break the $\mathrm{ON}-\mathrm{Cl}$ bond. Its extinction coefficient in the ultra-violet below $3350 \AA$. increases towards a maximum at around $1970 \AA .4$ and, under the conditions obtaining in the experi. ments reported here, most of the absorption occurs 